\title{
THE METRIC APPROACH TO FIXED POINT THEOREMS IN METRIC-LIKE SPACES
}

\author{
N. V. DUNG, N. T. HIEU, AND V. T. L. HANG
}

Received 18 December, 2015

\begin{abstract}
By constructing two metrics from a given metric-like space we show that some fixed point theorems in metric-like spaces may be obtained from the corresponding results in metric spaces.
\end{abstract}

2010 Mathematics Subject Classification: 47H10; 54H25; 54D99; 54E99

Keywords: fixed point, metric-like space

\section{INTRODUCTION AND PRELIMINARIES}

In 1992 Matthews [18] introduced the notion of a partial metric space as a part of the study of denotational semantics of data flow networks. The main difference comparing to the standard metric is that the self-distance of an arbitrary point need not be equal to zero. After that, partial metric spaces were investigated by many authors, see [14, 19, 28], [16, Chapter 14] and the references given there.

In 2012 Harandi [9] introduced the notion of a metric-like space as a generalization of a partial metric space and initiated the fixed point theory in this space. As an application, the author derived some new fixed point theorems in partial metric spaces. Note that a metric-like was mentioned before as a dislocated metric in [10,29].

After that metric-like spaces and its modifications were investigated by many others. Isik and Turkoglu [11] established some fixed point theorems for weakly contractive maps defined in ordered metric-like spaces. Malhotra et al. [17] obtained the fixed point results for $F$-type contractions which satisfy a weaker condition than the monotonicity of self-map of a partially ordered metric-like space. A fixed point result for $F$-expansive maps was also proved. Shukla et al. [25] introduced the notion of a $0-\sigma$-complete metric-like space and proved some common fixed point theorems in such space. Shobkolaei et al. [25] demonstrated a fundamental lemma for the convergence of sequences in metric-like spaces, and by using it authors proved some Suzuki-type fixed point results. Karapinar and Salimi [15] discussed the existence and uniqueness of a fixed point of a cyclic map in the context of metric-like spaces. Recently, Salimi et al. [23] introduced the notions of $\alpha-\psi \phi$-contractive and cyclic 
$\alpha-\psi \phi$-contractive maps and established the existence and uniqueness of fixed points for such maps in complete metric-like spaces. The results generalized and extended several comparable results in the existing literature.

The above results unified and generalized some well-known fixed point results in the literature. To prove these results, authors used similar techniques as that used to prove fixed point theorems in metric spaces.

In this paper we present another approach to fixed point theorems in metric-like spaces. By introducing two metrics induced from a metric-like space we show that some fixed point theorems in metric-like spaces may be deduced immediately from fixed point theorems in metric spaces. In particular, we show that

(1) [9, Theorem 2.7] is a consequence of [22, Theorem 1].

(2) [9, Theorem 2.11] is a consequence of [21, Corollary on page 463].

(3) [11, Theorem 1], [11, Theorem 2] and [11, Theorem 3] are consequences of [20, Corollary 3.3] and [20, Theorem 4.3].

(4) [25, Theorem 9] is a consequence of [27, Theorem 2].

(5) Various fixed point theorems in metric-like spaces in [1,2, 4, 5, 12, 13, 15, 17, $23,26]$ may be deduced from certain fixed point theorems in metric spaces by using similar arguments.

This work may be seen as a continuation of $[3,8]$ and some others in obtaining fixed point theorems on generalized metric spaces from fixed point theorems on certain metric spaces.

First, we recall some notions and lemmas which will be used latter.

Definition 1 ([9, Definition 2.1]). Let $X$ be a non-empty set and $\sigma: X \times X \longrightarrow \mathbb{R}^{+}$ be a function such that for all $x, y, z \in X$,

(1) If $\sigma(x, y)=0$ then $x=y$;

(2) $\sigma(x, y)=\sigma(y, x)$;

(3) $\sigma(x, z) \leq \sigma(x, y)+\sigma(y, z)$.

Then $\sigma$ is called a metric-like on $X$ and the pair $(X, \sigma)$ is called a metric-like space. Each metric-like $\sigma$ on $X$ generates a topology $\tau_{\sigma}$ on $X$ whose base is the family of open $\sigma$-balls

$$
B_{\sigma}(x, \varepsilon)=\{y \in X:|\sigma(x, y)-\sigma(x, x)|<\varepsilon\}
$$

for all $x \in X$ and $\varepsilon>0$. A sequence $\left\{x_{n}\right\}$ in $(X, \sigma)$ is called convergent to a point $x \in X$ if $\lim _{n \rightarrow \infty} \sigma\left(x_{n}, x\right)=\sigma(x, x)$, written $\lim _{n \rightarrow \infty} x_{n}=x$. A sequence $\left\{x_{n}\right\}$ in $(X, \sigma)$ is called $\sigma$-Cauchy if $\lim _{n, m \rightarrow \infty} \sigma\left(x_{n}, x_{m}\right)$ exists and is finite. The metric-like space $(X, \sigma)$ is called complete if for each $\sigma$-Cauchy sequence $\left\{x_{n}\right\}$, there exists some $x \in X$ such that

$$
\lim _{n \rightarrow \infty} \sigma\left(x_{n}, x\right)=\sigma(x, x)=\lim _{n, m \rightarrow \infty} \sigma\left(x_{m}, x_{n}\right) .
$$


Remark 1 ([9, page 2]). (1) If $f: X \longrightarrow Y$ is a continuous map from a metriclike space $X$ to a metric-like space $Y$ and $\lim _{n \rightarrow \infty} x_{n}=x$ in $X$ then $\lim _{n \rightarrow \infty} f\left(x_{n}\right)$ $=f(x)$ in $Y$.

(2) Every partial metric space [6, Definition 2] is a metric-like space.

Some interesting examples of metric-like spaces are as follows.

Example 1 ([9, Example 2.2]). Let $X=\{0,1\}$ and

$$
\sigma(x, y)= \begin{cases}2 & \text { if } x=y \\ 1 & \text { if } x \neq y .\end{cases}
$$

Then $\sigma$ is a metric-like. Since $\sigma(0,0)>\sigma(0,1), \sigma$ is not a partial metric.

Example 2 ([17, Example 3]). Let $X=\mathbb{R}^{+}$and

$$
\sigma(x, y)= \begin{cases}2 x & \text { if } x=y \\ \max \{x, y\} & \text { if } x \neq y\end{cases}
$$

Then $\sigma$ is a metric-like. Since $\sigma(1,1)>\sigma(0,1), \sigma$ is not a partial metric.

\section{Metrics induCED by A MetriC-LiKe}

First, we present some new notions and lemmas in metric-like spaces.

Definition 2. Let $(X, \sigma)$ be a metric-like space.

(1) A sequence $\left\{x_{n}\right\}$ is called 0 - $\sigma$-Cauchy if $\lim _{n, m \rightarrow \infty} \sigma\left(x_{n}, x_{m}\right)=0$.

(2) $(X, \sigma)$ is called 0 -complete if for each $0-\sigma$-Cauchy sequence $\left\{x_{n}\right\}$, there exists some $x \in X$ such that

$$
\lim _{n \rightarrow \infty} \sigma\left(x_{n}, x\right)=\sigma(x, x)=\lim _{n, m \rightarrow \infty} \sigma\left(x_{m}, x_{n}\right)=0 .
$$

Lemma 1. Let $(X, \sigma)$ be a metric-like space.

(1) If $(X, \sigma)$ is complete then it is 0-complete.

(2) If $\lim _{n \rightarrow \infty} x_{n}=x, \lim _{n \rightarrow \infty} y_{n}=y$ and $\sigma(x, x)=\sigma(y, y)=0$ then $\lim _{n \rightarrow \infty} \sigma\left(x_{n}, y_{n}\right)$ $=\sigma(x, y)$.

Proof. (1). It is straightforward from the definition.

(2). The inequality

$$
\sigma\left(x_{n}, y_{n}\right) \leq \sigma\left(x_{n}, x\right)+\sigma(x, y)+\sigma\left(y, y_{n}\right)
$$

implies

Similarly

$$
\sigma\left(x_{n}, y_{n}\right)-\sigma(x, y) \leq \sigma\left(x_{n}, x\right)+\sigma\left(y, y_{n}\right) .
$$

implies

$$
\sigma(x, y) \leq \sigma\left(x, x_{n}\right)+\sigma\left(x_{n}, y_{n}\right)+\sigma\left(y_{n}, y\right)
$$

$$
\sigma(x, y)-\sigma\left(x_{n}, y_{n}\right) \leq \sigma\left(x_{n}, x\right)+\sigma\left(y, y_{n}\right) .
$$


So that

$$
\left|\sigma\left(x_{n}, y_{n}\right)-\sigma(x, y)\right| \leq \sigma\left(x_{n}, x\right)+\sigma\left(y_{n}, y\right) .
$$

Taking the limit as $n \rightarrow \infty$ in (2.1) and note that $\sigma(x, x)=\sigma(y, y)=0$, we have $\lim _{n \rightarrow \infty} \sigma\left(x_{n}, y_{n}\right)=\sigma(x, y)$.

The following example shows that the implication (1) in Lemma 1 can not be reversed.

Example 3. Let $X=(0,1)$ and $\sigma(x, y)=|x-y|+1$ for all $x, y \in X$. Then we have

(1) $\sigma$ is a metric-like.

(2) $(X, \sigma)$ is 0 -complete.

(3) $(X, \sigma)$ is not complete.

Proof. (1). It is easy to check that $\sigma$ is a metric-like on $X$.

(2). Since $\sigma(x, y) \geq 1$ for all $x, y \in X,(X, \sigma)$ has no any 0 - $\sigma$-Cauchy sequence. This shows that $(X, \sigma)$ is 0 -complete.

(3). We have that

$$
\lim _{n, m \rightarrow \infty} \sigma\left(\frac{1}{2 n}, \frac{1}{2 m}\right)=\lim _{n, m \rightarrow \infty}\left(\left|\frac{1}{2 n}-\frac{1}{2 m}\right|+1\right)=1 .
$$

Then $\left\{\frac{1}{2 n}\right\}$ is a $\sigma$-Cauchy sequence in $(X, \sigma)$. On the contrary, suppose that $\lim _{n \rightarrow \infty} \frac{1}{2 n}$ $=x$ in $(X, \sigma)$. Then

$$
\lim _{n \rightarrow \infty} \sigma\left(\frac{1}{2 n}, x\right)=\lim _{n \rightarrow \infty}\left(\left|\frac{1}{2 n}-x\right|+1\right)=\sigma(x, x)=1 .
$$

This implies that $x=0 \in X=(0,1)$. It is a contradiction. Then $\left\{\frac{1}{2 n}\right\}$ is not convergent in $(X, \sigma)$. Therefore, $(X, \sigma)$ is not complete.

From a given metric-like we construct two metrics as follows.

Theorem 1. Let $(X, \sigma)$ be a metric-like space and

$$
\begin{gathered}
d_{\sigma}(x, y)= \begin{cases}0 & \text { if } x=y \\
\sigma(x, y) & \text { if } x \neq y\end{cases} \\
m_{\sigma}(x, y)= \begin{cases}0 & \text { if } x=y \\
\max \{\sigma(x, y), \sigma(x, x), \sigma(y, y)\} & \text { if } x \neq y .\end{cases}
\end{gathered}
$$

Then we have

(1) $d_{\sigma}$ and $m_{\sigma}$ are metrics on $X$.

(2) The metric-like space $(X, \sigma)$ is 0-complete if and only if the metric space $\left(X, d_{\sigma}\right)$ is complete.

(3) The metric-like space $(X, \sigma)$ is 0-complete if and only if the metric space $\left(X, m_{\sigma}\right)$ is complete. 
Proof. (1). It is clear that $d_{\sigma}$ is a metric on $X$. For all $x, y \in X$, we have $m_{\sigma}(x, y)=0$ if and only if $x=y$ and $m_{\sigma}(x, y)=m_{\sigma}(y, x)$. Now, for all $x, y, z \in X$, we may assume $x \neq y \neq z$. Then

$$
\begin{aligned}
m_{\sigma}(x, z) & =\max \{\sigma(x, z), \sigma(x, x), \sigma(z, z)\} \\
& \leq \max \{\sigma(x, y)+\sigma(y, z), \sigma(x, x), \sigma(z, z)\} \\
& \leq \max \{\sigma(x, y), \sigma(x, x)\}+\max \{\sigma(y, z), \sigma(z, z)\} \\
& \leq \max \{\sigma(x, y), \sigma(x, x), \sigma(y, y)\}+\max \{\sigma(y, z), \sigma(y, y), \sigma(z, z)\} \\
& =m_{\sigma}(x, y)+m_{\sigma}(y, z) .
\end{aligned}
$$

Therefore, $m_{\sigma}$ is also a metric on $X$.

(2). Suppose that $(X, \sigma)$ is 0 -complete and let $\left\{x_{n}\right\}$ be a Cauchy sequence in $\left(X, d_{\sigma}\right)$.

If there exists $x \in X$ such that the set $\left\{n \in \mathbb{N}: x_{n}=x\right\}$ is infinite then we get a subsequence $\left\{x_{n_{k}}\right\}$ of $\left\{x_{n}\right\}$ such that $d_{\sigma}\left(x_{n_{k}}, x\right)=0$ for all $k \in \mathbb{N}$. So $\lim _{k \rightarrow \infty} x_{n_{k}}=x$ in $\left(X, d_{\sigma}\right)$. Since $\left\{x_{n}\right\}$ is a Cauchy sequence in $\left(X, d_{\sigma}\right)$, we get $\lim _{n \rightarrow \infty} x_{n}=x$ in $\left(X, d_{\sigma}\right)$.

If this situation does not happen then there exists $n_{0} \in \mathbb{N}$ such that $x_{n} \neq x_{m}$ for all $n \neq m \geq n_{0}$. So $\sigma\left(x_{n}, x_{m}\right)=d_{\sigma}\left(x_{n}, x_{m}\right)$ for all $n \neq m \geq n_{0}$. In this case $\lim _{n, m \rightarrow \infty} \sigma\left(x_{n}, x_{m}\right)=\lim _{n, m \rightarrow \infty} d_{\sigma}\left(x_{n}, x_{m}\right)=0$. Then $\left\{x_{n}\right\}$ is a $0-\sigma$-Cauchy sequence. By hypothesis there exists $x \in X$ such that

$$
\lim _{n \rightarrow \infty} \sigma\left(x_{n}, x\right)=\sigma(x, x)=\lim _{n, m \rightarrow \infty} \sigma\left(x_{n}, x_{m}\right)=0 .
$$

Then $\lim _{n \rightarrow \infty} d_{\sigma}\left(x_{n}, x\right)=\lim _{n \rightarrow \infty} \sigma\left(x_{n}, x\right)=0$. So $\lim _{n \rightarrow \infty} x_{n}=x$ in $\left(X, d_{\sigma}\right)$.

By the above, the metric space $\left(X, d_{\sigma}\right)$ is complete.

Conversely, suppose that $\left(X, d_{\sigma}\right)$ is complete and let $\left\{x_{n}\right\}$ be a $0-\sigma$-Cauchy sequence in the metric-like space $(X, \sigma)$. Then

$$
d_{\sigma}\left(x_{n}, x_{m}\right)= \begin{cases}\sigma\left(x_{n}, x_{m}\right) & \text { if } x_{n} \neq x_{m} \\ 0 & \text { if } x_{n}=x_{m}\end{cases}
$$

for all $n, m \in \mathbb{N}$. This implies that $\left\{x_{n}\right\}$ is a Cauchy sequence in $\left(X, d_{\sigma}\right)$. So there exists $x \in X$ such that $\lim _{n \rightarrow \infty} x_{n}=x$ in $\left(X, d_{\sigma}\right)$.

If the set $M_{x}=\left\{n \in \mathbb{N}: x_{n}=x\right\}$ is infinite then we get a subsequence $\left\{x_{n_{k}}\right\}$ of $\left\{x_{n}\right\}$ such that $\sigma\left(x_{n_{k}}, x_{m_{k}}\right)=\sigma(x, x)$ for all $k$. Since $\left\{x_{n}\right\}$ is $0-\sigma$-Cauchy, we get $\lim _{k \rightarrow \infty} \sigma\left(x_{n_{k}}, x_{m_{k}}\right)=\sigma(x, x)=0$. So $\lim _{k \rightarrow \infty} \sigma\left(x_{n_{k}}, x\right)=\sigma(x, x)=0$. Note that $\sigma\left(x_{k}, x\right) \leq \sigma\left(x_{k}, x_{n_{k}}\right)+\sigma\left(x_{n_{k}}, x\right)$ for all $k \in \mathbb{N}$ and $\left\{x_{n}\right\}$ is a $0-\sigma$-Cauchy in $(X, \sigma)$. So $\lim _{k \rightarrow \infty} \sigma\left(x_{k}, x\right)=0$. This shows that $\lim _{n \rightarrow \infty} x_{n}=x$ in $(X, \sigma)$. For $n \in \mathbb{N} \backslash M_{x}$ we get $x_{n} \neq x$ and so $\sigma\left(x_{n}, x\right)=d_{\sigma}\left(x_{n}, x\right)$. This implies that $\lim _{n \rightarrow \infty} \sigma\left(x_{n}, x\right)=$ 
$\lim _{n \rightarrow \infty} d_{\sigma}\left(x_{n}, x\right)=0$. Note that

$$
0 \leq \sigma(x, x) \leq \lim _{n \rightarrow \infty}\left[\sigma\left(x, x_{n}\right)+\sigma\left(x_{n}, x\right)\right]=0 .
$$

Then we have

$$
\lim _{n \rightarrow \infty} \sigma\left(x_{n}, x\right)=\sigma(x, x)=\lim _{n, m \rightarrow \infty} \sigma\left(x_{n}, x_{m}\right)=0 .
$$

By the above, the metric-like space $(X, \sigma)$ is 0-complete.

(3). Suppose that $(X, \sigma)$ is 0 -complete and let $\left\{x_{n}\right\}$ be a Cauchy sequence in the metric space $\left(X, m_{\sigma}\right)$. If there exists $x \in X$ such that $\left\{n \in \mathbb{N}: x_{n}=x\right\}$ is infinite then, as in the proof of (2), it follows $\lim _{n \rightarrow \infty} x_{n}=x$ in $\left(X, m_{\sigma}\right)$.

Suppose now that $x_{n} \neq x_{m}$ for all $n \neq m \in \mathbb{N}$. Then we have

$$
\begin{aligned}
0 \leq \lim _{n, m \rightarrow \infty} \sigma\left(x_{n}, x_{m}\right) & \leq \lim _{n, m \rightarrow \infty} \max \left\{\sigma\left(x_{n}, x_{m}\right), \sigma\left(x_{n}, x_{n}\right), \sigma\left(x_{m}, x_{m}\right)\right\} \\
& =\lim _{n, m \rightarrow \infty} m_{\sigma}\left(x_{n}, x_{m}\right)=0 .
\end{aligned}
$$

This shows that $\left\{x_{n}\right\}$ is a $0-\sigma$-Cauchy sequence in the 0 -complete metric-like space $(X, \sigma)$. Then there exists $x \in X$ such that

$$
\lim _{n \rightarrow \infty} \sigma\left(x_{n}, x\right)=\sigma(x, x)=\lim _{n, m \rightarrow \infty} \sigma\left(x_{n}, x_{m}\right)=0 .
$$

It follows that $\lim _{n \rightarrow \infty} \sigma\left(x_{n}, x_{n}\right)=0$ and so

$$
\lim _{n \rightarrow \infty} m_{\sigma}\left(x_{n}, x\right)=\lim _{n \rightarrow \infty} \max \left\{\sigma\left(x_{n}, x\right), \sigma\left(x_{n}, x_{n}\right), \sigma(x, x)\right\}=0 .
$$

This shows that $\lim _{n \rightarrow \infty} x_{n}=x$ in $\left(X, m_{\sigma}\right)$.

By the above, the metric space $\left(X, m_{\sigma}\right)$ is complete.

Conversely, suppose that $\left(X, m_{\sigma}\right)$ is complete and let $\left\{x_{n}\right\}$ be a $0-\sigma$-Cauchy sequence in the metric-like space $(X, \sigma)$. So $\lim _{n, m \rightarrow \infty} \sigma\left(x_{n}, x_{m}\right)=0$. Then

$$
\lim _{n \rightarrow \infty} \sigma\left(x_{n}, x_{n}\right)=\lim _{m \rightarrow \infty} \sigma\left(x_{m}, x_{m}\right)=0 .
$$

So that

$$
\lim _{n, m \rightarrow \infty} m_{\sigma}\left(x_{n}, x_{m}\right)=\lim _{n, m \rightarrow \infty} \max \left\{\sigma\left(x_{n}, x_{m}\right), \sigma\left(x_{n}, x_{n}\right), \sigma\left(x_{m}, x_{m}\right)\right\}=0 .
$$

Thus $\left\{x_{n}\right\}$ is a Cauchy sequence in the complete metric space $\left(X, m_{\sigma}\right)$. Then there exists $x \in X$ such that

$$
\lim _{n \rightarrow \infty} m_{\sigma}\left(x_{n}, x\right)=\lim _{n \rightarrow \infty} \max \left\{\sigma\left(x_{n}, x\right), \sigma\left(x_{n}, x_{n}\right), \sigma(x, x)\right\}=0 .
$$

It implies that $\lim _{n \rightarrow \infty} \sigma\left(x_{n}, x\right)=\sigma(x, x)=0$. Therefore,

$$
\lim _{n \rightarrow \infty} \sigma\left(x_{n}, x\right)=\sigma(x, x)=\lim _{n, m \rightarrow \infty} \sigma\left(x_{n}, x_{m}\right)=0 .
$$


So $\lim _{n \rightarrow \infty} x_{n}=x$ in $(X, \sigma)$. This shows that the metric-like space $(X, \sigma)$ is 0 -complete.

Next, we prove a technical lemma on the relation between certain values relating the metric-like space $(X, \sigma)$ and the metric space $\left(X, m_{\sigma}\right)$ mentioned in Theorem 1 .

Lemma 2. Let $(X, \sigma)$ be a metric-like space, $T: X \longrightarrow X$ be a map and for all $x, y \in X$,

$$
\begin{gathered}
M_{\sigma}(x, y)=\max \{\sigma(x, y), \sigma(x, T x), \sigma(y, T y), \sigma(x, T y), \sigma(y, T x), \sigma(x, x), \\
\sigma(y, y), \sigma(T x, T x), \sigma(T y, T y)\}, \\
M_{m_{\sigma}}(x, y)=\max \left\{m_{\sigma}(x, y), m_{\sigma}(x, T x), m_{\sigma}(y, T y), m_{\sigma}(x, T y), m_{\sigma}(y, T x)\right\} .
\end{gathered}
$$

Then $M_{\sigma}(x, y)=M_{m_{\sigma}}(x, y)$ for all $x \neq y \in X$.

Proof. We first show that $M_{\sigma}(x, y) \leq M_{m_{\sigma}}(x, y)$ for all $x \neq y \in X$. We only consider the following four cases.

Case 1. $M_{\sigma}(x, y)=\max \{\sigma(x, y), \sigma(x, x), \sigma(y, y)\}$. Then

$$
M_{\sigma}(x, y)=m_{\sigma}(x, y) \leq M_{m_{\sigma}}(x, y) .
$$

Case 2. $M_{\sigma}(x, y)=\sigma(x, T x)$. If $T x=x$ then

$$
M_{\sigma}(x, y)=\sigma(x, x) \leq m_{\sigma}(x, y) \leq M_{m_{\sigma}}(x, y) .
$$

If $T x \neq x$ then

$$
\begin{aligned}
M_{\sigma}(x, y)=\sigma(x, T x) \leq \max & \{\sigma(x, T x), \sigma(x, x), \sigma(T x, T x)\}=m_{\sigma}(x, T x) \\
& \leq M_{m_{\sigma}}(x, y) .
\end{aligned}
$$

Case 3. $M_{\sigma}(x, y)=\sigma(x, T y)$. If $T y=x$ then

$$
M_{\sigma}(x, y)=\sigma(x, x) \leq m_{\sigma}(x, y) \leq M_{m_{\sigma}}(x, y) .
$$

If $T y \neq x$ then

$$
\begin{aligned}
M_{\sigma}(x, y)=\sigma(x, T y) \leq \max & \{\sigma(x, T y), \sigma(x, x), \sigma(T y, T y)\}=m_{\sigma}(x, T y) \\
& \leq M_{m_{\sigma}}(x, y) .
\end{aligned}
$$

Case 4. $M_{\sigma}(x, y)=\sigma(T x, T x)$. If $T x=x$ then

$$
M_{\sigma}(x, y)=\sigma(x, x) \leq m_{\sigma}(x, y) \leq M_{m_{\sigma}}(x, y) .
$$

If $T x \neq x$ then

$$
\begin{aligned}
M_{\sigma}(x, y)=\sigma(T x, T x) \leq \max & \{\sigma(x, T x), \sigma(x, x), \sigma(T x, T x)\}=m_{\sigma}(x, T x) \\
& \leq M_{m_{\sigma}}(x, y) .
\end{aligned}
$$

We next show that $M_{\sigma}(x, y) \geq M_{m_{\sigma}}(x, y)$ for all $x \neq y \in X$. We only consider the following three cases. 
Case 1. $M_{m_{\sigma}}(x, y)=m_{\sigma}(x, y)$. Then

$$
M_{m_{\sigma}}(x, y)=\max \{\sigma(x, y), \sigma(x, x), \sigma(y, y)\} \leq M_{\sigma}(x, y) .
$$

Case 2. $M_{m_{\sigma}}(x, y)=m_{\sigma}(x, T x)$. If $T x=x$ then

$$
M_{m_{\sigma}}(x, y)=0 \leq M_{\sigma}(x, y) .
$$

If $T x \neq x$ then

$$
M_{m_{\sigma}}(x, y)=m_{\sigma}(x, T x)=\max \{\sigma(x, T x), \sigma(x, x), \sigma(T x, T x)\} \leq M_{\sigma}(x, y) .
$$

Case 3. $M_{m_{\sigma}}(x, y)=m_{\sigma}(x, T y)$. If $T y=x$ then

$$
M_{m_{\sigma}}(x, y)=0 \leq M_{\sigma}(x, y) .
$$

If $T y \neq x$ then

$$
M_{m_{\sigma}}(x, y)=m_{\sigma}(x, T y)=\max \{\sigma(x, T y), \sigma(x, x), \sigma(T y, T y)\} \leq M_{\sigma}(x, y) .
$$

By the above, we have $M_{\sigma}(x, y)=M_{m_{\sigma}}(x, y)$.

\section{ApplicAtions}

In this section we show that some fixed point theorems in metric-like spaces may be obtained from the corresponding results in metric spaces. The following results are consequences of [7, Theorem 2.1].

Theorem 2. Let $(X, \sigma)$ be a complete metric-like space and $T: X \longrightarrow X$ be a map such that

$$
\sigma(T x, T y) \leq \psi\left(M_{\sigma}(x, y)\right)
$$

for all $x, y \in X$, where $M_{\sigma}(x, y)$ is defined as in Lemma 2 , and $\psi:[0, \infty) \longrightarrow[0, \infty)$ is $a$ non-decreasing function satisfying $\psi(t)<t, \lim _{s \rightarrow t^{+}} \psi(s)$ $<t$ for all $t>0$ and $\lim _{t \rightarrow \infty}[t-\psi(t)]=\infty$. Then $T$ has a fixed point .

Proof. Since $\psi$ is non-decreasing, it follows from (3.1) that

$$
\sigma(T x, T x) \leq \psi(\max \{\sigma(x, x), \sigma(x, T x), \sigma(T x, T x)\}) \leq \psi\left(M_{\sigma}(x, y)\right)
$$

for all $x, y \in X$. By using (3.1), (3.2) and Lemma 2, we have, for $T x \neq T y$,

$$
\begin{aligned}
m_{\sigma}(T x, T y) & =\max \{\sigma(T x, T y), \sigma(T x, T x), \sigma(T y, T y)\} \leq \psi\left(M_{\sigma}(x, y)\right) \\
& =\psi\left(M_{m_{\sigma}}(x, y)\right) .
\end{aligned}
$$

Note that if $T x=T y$ then the inequality $m_{\sigma}(T x, T y) \leq \psi\left(M_{m_{\sigma}}(x, y)\right)$ is obviously true. Then, from Lemma 1.(1) and Theorem 1.(3), we obtain that $\left(X, m_{\sigma}\right)$ is a complete metric space. Then, by using [7, Theorem 2.1], we get the conclusion. 
Theorem 3 ([9, Theorem 2.4]). Let $(X, \sigma)$ be a complete metric-like space and $T: X \longrightarrow X$ be a map such that

$$
\sigma(T x, T y) \leq \psi(M(x, y))
$$

for all $x, y \in X$, where

$$
\begin{aligned}
& M(x, y)=\max \{\sigma(x, y), \sigma(x, T x), \sigma(y, T y), \sigma(x, T y), \sigma(y, T x), \sigma(x, x), \sigma(y, y)\} \\
& \text { and } \psi:[0, \infty) \longrightarrow[0, \infty) \text { is a non-decreasing function satisfying } \psi(t)<t, \lim _{s \rightarrow t^{+}} \psi(s) \\
& <t \text { for all } t>0 \text { and } \lim _{t \rightarrow \infty}[t-\psi(t)]=\infty . \text { Then } T \text { has a fixed point. }
\end{aligned}
$$

Proof. Since $M(x, y) \leq M_{\sigma}(x, y)$ for all $x, y \in X$ and $\psi$ is non-decreasing, we see that (3.3) implies (3.1). Then the conclusion is a direct consequence of Theorem 2.

The following is a consequence of [22, Theorem 1].

Theorem 4 ([9, Theorem 2.7]). Let $(X, \sigma)$ be a complete metric-like space and $T: X \longrightarrow X$ be a map such that

$$
\sigma(T x, T y) \leq \sigma(x, y)-\varphi(\sigma(x, y))
$$

for all $x, y \in X$, where $\varphi:[0, \infty) \longrightarrow[0, \infty)$ is a non-decreasing continuous function satisfying $\varphi(t)=0$ if and only if $t=0$. Then $T$ has a unique fixed point.

Proof. We will prove that

$$
d_{\sigma}(T x, T y) \leq d_{\sigma}(x, y)-\varphi\left(d_{\sigma}(x, y)\right) .
$$

by considering the following three cases.

Case 1. $T x \neq T y$. Then (3.5) is a direct consequence of (3.4).

Case 2. $T x=T y$ and $x=y$. Then (3.5) becomes $0 \leq 0-\varphi(0)$ and that obviously holds.

Case 3. $T x=T y$ and $x \neq y$. Then (3.5) becomes $0 \leq \sigma(x, y)-\varphi(\sigma(x, y))$ and that holds by (3.4).

By the above cases, we have that (3.5) holds for all $x, y \in X$. From Lemma 1.(1) and Theorem 1.(2), we obtain that $\left(X, d_{\sigma}\right)$ is a complete metric space. Then, by using [22, Theorem 1], we get the conclusion.

The following result is a consequence of [21, Corollary on page 463].

Theorem 5 ([9, Theorem 2.11]). Let $(X, \sigma)$ be a complete metric-like space and $T: X \longrightarrow X$ be a map such that

$$
\sigma(T x, T y) \leq \alpha(\sigma(x, y)) \sigma(x, y)
$$

for all $x, y \in X$, where $\alpha:[0, \infty) \longrightarrow[0, \infty)$ is non-increasing. Then $T$ has a unique fixed point. 
Proof. Using [21, Corollary on page 463] and the argument as in the proof of Theorem 4.

Remark 2. There exist a metric-like space $(X, \sigma)$ and a map $T: X \longrightarrow X$ such that

$$
\begin{aligned}
& \max \left\{\sigma\left(x_{n}, x_{n-1}\right), \sigma\left(x_{n+1}, x_{n}\right)\right\} \\
<\max & \left\{\sigma\left(x_{n}, x_{n-1}\right), \sigma\left(x_{n}, T x_{n}\right), \sigma\left(x_{n-1}, T x_{n-1}\right), \sigma\left(x_{n}, x_{n}\right), \sigma\left(x_{n-1}, x_{n-1}\right),\right. \\
& \left.\frac{1}{2}\left[\sigma\left(x_{n}, T x_{n-1}\right)+\sigma\left(T x_{n}, x_{n-1}\right)\right]\right\}
\end{aligned}
$$

where $x_{n}=T x_{n-1}$ for all $n \in \mathbb{N}$ and all $x_{0} \in X$.

Proof. Let $X=\{1,2\}$ and $\sigma(1,1)=\sigma(2,2)=1, \sigma(1,2)=\sigma(2,1)=\frac{1}{2}$. Then $(X, \sigma)$ is a metric-like space. Put $T 1=2, T 2=1$. Then we have

$$
\begin{aligned}
\frac{1}{2} & =\max \left\{\sigma\left(x_{n}, x_{n-1}\right), \sigma\left(x_{n+1}, x_{n}\right)\right\} \\
& <1 \\
& =\max \left\{\sigma\left(x_{n}, x_{n-1}\right), \sigma\left(x_{n}, T x_{n}\right), \sigma\left(x_{n-1}, T x_{n-1}\right), \sigma\left(x_{n}, x_{n}\right), \sigma\left(x_{n-1}, x_{n-1}\right),\right. \\
& \left.\quad \frac{1}{2}\left[\sigma\left(x_{n}, T x_{n-1}\right)+\sigma\left(T x_{n}, x_{n-1}\right)\right]\right\} .
\end{aligned}
$$

Remark 2 implies the argument on [11, page 4] that

$$
\begin{aligned}
& \max \left\{\sigma\left(x_{n}, x_{n-1}\right), \sigma\left(x_{n}, T x_{n}\right), \sigma\left(x_{n-1}, T x_{n-1}\right), \sigma\left(x_{n}, x_{n}\right), \sigma\left(x_{n-1}, x_{n-1}\right),\right. \\
& \left.\quad \frac{1}{2}\left[\sigma\left(x_{n}, T x_{n-1}\right)+\sigma\left(T x_{n}, x_{n-1}\right)\right]\right\} \\
& \leq \max \left\{\sigma\left(x_{n}, x_{n-1}\right), \sigma\left(x_{n+1}, x_{n}\right)\right\}
\end{aligned}
$$

is not correct. Then [11, Theorem 1], [11, Theorem 2] and [11, Theorem 3] must to be restated as in Theorem 6, Theorem 7 and Theorem 8 respectively, where

$$
\max \left\{\sigma(x, y), \sigma(x, T x), \sigma(y, T y), \sigma(x, x), \sigma(y, y), \frac{1}{2}[\sigma(x, T y)+\sigma(y, T x)]\right\}
$$

in mentioned theorems is replaced by

$$
\max \left\{\sigma(x, y), \sigma(x, T x), \sigma(y, T y), \frac{1}{2}[\sigma(x, T y)+\sigma(y, T x)]\right\} .
$$

Note that they are consequences of [20, Corollary 3.3] and [20, Theorem 4.3].

Theorem 6. Let $(X, \preceq, \sigma)$ be a partially ordered complete metric-like space and $T: X \longrightarrow X$ be a continuous and non-decreasing map such that for all comparable $x, y \in X$,

$$
\psi(\sigma(T x, T y)) \leq \psi(N(x, y))-\phi(N(x, y))
$$


where

$$
N(x, y)=\max \left\{\sigma(x, y), \sigma(x, T x), \sigma(y, T y), \frac{1}{2}[\sigma(x, T y)+\sigma(y, T x)]\right\}
$$

and

(1) $\psi:[0, \infty) \longrightarrow[0, \infty)$ is continuous non-decreasing function with $\psi(t)=0$ if and only if $t=0$;

(2) $\phi:[0, \infty) \longrightarrow[0, \infty)$ is a lower semi-continuous function with $\phi(t)=0$ if and only if $t=0$.

If there exists $x_{0} \in X$ with $x_{0} \preceq T x_{0}$ then $T$ has a fixed point.

Proof. Using [20, Corollary 3.3] and the argument as in the proof of Theorem 4.

Theorem 7. Under the same hypotheses of Theorem 6 and without assuming the continuity of $T$, assume that whenever $\left\{x_{n}\right\}$ is a non-decreasing sequence in $X$ such that $\lim _{n \rightarrow \infty} x_{n}=x$ implies $x_{n} \preceq x$ for all $n \in \mathbb{N}$, then $T$ has a fixed point in $X$.

Proof. By using [20, Corollary 3.3] and the argument as in the proof of Theorem 4.

Theorem 8. Let all conditions of Theorem 6 (resp. Theorem 7) be fulfilled and let the following condition be satisfied: for arbitrary two points $x, y \in X$, there exists $z \in X$ which is comparable with both $x$ and $y$. Then the fixed point of $T$ is unique.

Proof. Using [20, Theorem 4.3] and the argument as in the proof of Theorem 4.

Theorem 9 ([25, Theorem 9]). Let $(X, \sigma)$ be a complete metric-like space, $\theta$ : $[0,1) \longrightarrow\left(\frac{1}{2}, 1\right]$ be a function defined by

$$
\theta(r)= \begin{cases}1 & \text { if } 0 \leq r \leq \frac{\sqrt{5}-1}{2} \\ \frac{1-r}{r^{2}} & \text { if } \frac{\sqrt{5}-1}{2} \leq r \leq \frac{1}{\sqrt{2}} \\ \frac{1}{1+r} & \text { if } \frac{\sqrt{2} \leq r \leq 1}{\sqrt{2}}\end{cases}
$$

and $T: X \longrightarrow X$ be a map such that for all $x, y \in X$,

$$
\theta(r) \sigma(x, T x) \leq \sigma(x, y) \text { implies } \sigma(T x, T y) \leq r \sigma(x, y) .
$$

Then $T$ has a unique fixed point $z \in X$ and for each $x \in X, \lim _{n \rightarrow \infty} T^{n} x=z$.

Proof. We will prove that for all $x, y \in X$,

$$
\theta(r) d_{\sigma}(x, T x) \leq d_{\sigma}(x, y) \text { implies } d_{\sigma}(T x, T y) \leq r d_{\sigma}(x, y) .
$$

If $T x=T y$ then $d_{\sigma}(T x, T y)=0$ and thus (3.9) obviously holds. If $T x \neq T y$ then $x \neq y$ and thus $d_{\sigma}(T x, T y)=\sigma(T x, T y), d_{\sigma}(x, y)=\sigma(x, y)$. Note that if 
$x=T x$ then $T$ obviously has fixed point. So we may assume that $x \neq T x$ and then $d_{\sigma}(x, T x)=\sigma(x, T x)$. In this case we find that (3.8) becomes (3.9).

So (3.9) holds for all $x, y \in X$. By Theorem 1 we see that $\left(X, d_{\sigma}\right)$ is complete since $(X, \sigma)$ is complete. By [27, Theorem 2] we get the conclusion.

Remark 3. By using similar arguments as in the proofs of above corollaries, we may obtain various fixed point theorems in metric-like spaces in $[1,2,4,5,12,13,15$, 17,23,26] from certain fixed point theorems in metric spaces. Then authors should be careful on stating fixed point theorems in metric-like spaces. Note that every partial metric space is a metric-like space and for more details on obtaining fixed point theorems on partial metric spaces from fixed point theorems in metric spaces, readers may refer to [8] and [24].

\section{ACKNOWLEDGEMENT}

The authors express their gratitude to anonymous reviewers for their helpful comments.

\section{REFERENCES}

[1] S. A. Al-Mezel, C. M. Chen, E. Karapinar, and V. Rakocević, "Fixed point results for various $\alpha$-admissible contractive mappings on metric-like spaces," Abstr. Appl. Anal., vol. 2014, pp. 1 15, 2014, doi: 10.1155/2014/379358.

[2] H. H. Alsulami, E. Karapinar, and H. Piri, "Fixed points of modified $F$-contractive mappings in complete metric-like spaces," J. Funct. Spaces, vol. 2015, pp. 1 - 9, 2015, doi: $10.1155 / 2015 / 270971$.

[3] T. V. An, N. V. Dung, and V. T. L. Hang, "A new approach to fixed point theorems on $G$-metric spaces,” Topology Appl., vol. 160, pp. 1486 - 1493, 2013, doi: 10.1016/j.topol.2013.05.027.

[4] H. Aydi, A. Felhi, and S. Sahmim, "Fixed points of multivalued nonself almost contractions in metric-like spaces," Math. Sci., vol. 9, no. 2, pp. 103 - 108, 2015, doi: 10.1007/s40096-015-01567.

[5] H. Aydi and E. Karapinar, "Fixed point results for generalized $\alpha-\psi$-contractions in metric-like spaces and applications," Electron. J. Differential Equations, vol. 2015, no. 133, pp. 1 - 15, 2015.

[6] M. Bukatin, R. Kopperman, S. Matthews, and H. Pajoohesh, "Partial metric spaces," Amer. Math. Monthly, vol. 116, pp. 708 - 718, 2009, doi: 10.4169/193009709X460831.

[7] L. Ćirić, N. Hussain, and N. Cakić, "Common fixed points for Ćirić type- $f$-weak contraction with application,” Publ. Math. Debrecen, vol. 76, no. 1-2, pp. 31 - 49, 2010.

[8] R. H. Haghi, S. Rezapour, and N. Shahzad, "Be careful on partial metric fixed point results," Topology Appl., vol. 160, no. 3, pp. 450 - 454, 2013, doi: 10.1016/j.topol.2012.11.004.

[9] A. A. Harandi, "Metric-like spaces, partial metric spaces and fixed points," Fixed Point Theory Appl., vol. 2012:204, pp. 1 - 11, 2012, doi: 10.1186/1687-1812-2012-204.

[10] P. Hitzler and A. K. Seda, "Dislocated topologies," J. Electr. Engin., vol. 51, pp. 3 - 7, 2000.

[11] H. Işik and D. Türkoglu, "Fixed point theorems for weakly contractive mappings in partially ordered metric-like spaces," Fixed Point Theory Appl., vol. 2013:51, pp. 1 - 11, 2013, doi: 10.1186/1687-1812-2013-51.

[12] M. Jleli, B. Samet, C. Vetro, and F. Vetro, "From Caristi's theorem to Ekeland's variational principle in $0_{\sigma}$-complete metric-like spaces," Abstr. Appl. Anal., vol. 2014, pp. 1 - 7, 2014, doi: 10.1155/2014/319619. 
[13] E. Karapinar, M. A. Kutbi, H. Piri, and D. O'Regan, "Fixed points of conditionally $F$-contractions in complete metric-like spaces," Fixed Point Theory Appl., vol. 2015:126, pp. 1 - 14, 2015.

[14] E. Karapinar, W. S. Du, P. Kumam, A. Petruşel, and S. Romaguera, Existence and uniqueness of fixed point in various abstract spaces and related applications. Hindawi Publishing Corporation, 2015. doi: 1.

[15] E. Karapinar and P. Salimi, "Dislocated metric space to metric spaces with some fixed point theorems," Fixed Point Theory Appl., vol. 2013:222, pp. 1 - 13, 2013, doi: 10.1186/1687-1812-2013222.

[16] W. Kirk and N. Shahzad, Fixed point theory in distance spaces. Cham: Springer, 2014. doi: 10.1007/978-3-319-10927-5.

[17] S. K. Malhotra, S. Radenovic, and S. Shukla, "Some fixed point results without monotone property in partially ordered metric-like spaces," J. Egyptian Math. Soc., vol. 22, pp. 83 - 89, 2014, doi: 10.1016/j.joems.2013.06.010.

[18] S. G. Matthews, Partial metric topology. Queen's College: Papers on general topology and applications, Proc. 8th Summer Conf., 1992. doi: 1.

[19] H. K. Nashine, Z. Kadelburg, and S. Radenović, "Fixed point theorems via various cyclic contractive conditions in partial metric spaces," Publ. Inst. Math. (Beograd)(N.S.), vol. 93, no. 107, pp. $69-93,2013$, doi: 10.2298/PIM1307069N.

[20] S. Radenović and Z. Kadelburg, "Generalized weak contractions in partially ordered metric spaces," Comput. Math. Appl., vol. 60, pp. 1776 - 1783, 2010, doi: 10.1016/j.cam.2015.05.017.

[21] E. Rakotch, "A note on contractive mappings," Proc. Amer. Math. Soc., vol. 13, no. 3, pp. $459-$ $465,1962$.

[22] B. E. Rhoades, "Some theorems on weakly contractive maps," Nonlinear Anal., vol. 47, no. 4, pp. 2683-2693, 2001, doi: 10.1016/S0362-546X(01)00388-1.

[23] P. Salimi, N. Hussain, S. Shukla, S. F. Fathollahi, and S. Radenović, "Fixed point results for cyclic $\alpha-\psi \phi$-contractions with application to integral equations," J. Comput. Appl. Math., vol. 290, pp. 445 - 458, 2015, doi: 10.1016/j.cam.2015.05.017.

[24] B. SametEmail, C. Vetro, and F. Vetro, "From metric spaces to partial metric spaces," Fixed Point Theory Appl., vol. 2013:5, pp. 1 - 11, 2013, doi: 10.1186/1687-1812-2013-5.

[25] N. Shobkolaei, S. Sedghi, J. R. Roshan, and N. Hussain, "Suzuki-type fixed point results in metriclike spaces," J. Funct. Spaces Appl., vol. 2013, pp. 1 - 9, 2013, doi: 10.1155/2013/143686.

[26] S. Shukla, S. Radenović, and V. C. Rajić, "Some common fixed point theorems in 0- $\sigma$-complete metric-like spaces," Vietnam J. Math., vol. 41, no. 3, pp. 341 - 352, 2013, doi: 0.1007/s10013013-0028-0.

[27] T. Suzuki, "A generalized Banach contraction principle that characterizes metric completeness," Proc. Amer. Math. Soc., vol. 136, pp. 1861 - 1869, 2008, doi: 10.1090/S0002-9939-07-09055-7.

[28] C. Vetroa and F. Vetro, "Metric or partial metric spaces endowed with a finite number of graphs: A tool to obtain fixed point results," Topology Appl., vol. 164, pp. 125 - 137, 2014, doi: 10.1016/j.topol.2013.12.008.

[29] P. Waszkiewicz, "The local triangle axioms in topology and domain theory," Appl. Gen. Topol., vol. 4, no. 1, pp. 47 - 70, 2003, doi: 10.4995/agt.2003.2009.

\section{Authors' addresses}

\section{N. V. Dung}

Nonlinear Analysis Research Group, Ton Duc Thang University, Ho Chi Minh City, Vietnam Faculty of Mathematics and Statistics, Ton Duc Thang University, Ho Chi Minh City, Vietnam E-mail address: nguyenvandung2etdt.edu.vn 


\section{N. T. Hieu}

Faculty of Mathematics and Information Technology Teacher Education, Dong Thap University, Cao Lanh City, Dong Thap Province, Vietnam

E-mail address: ngtrunghieuedthu.edu.vn

\section{T. L. Hang}

Journal of Science, Dong Thap University, Cao Lanh City, Dong Thap Province, Vietnam

E-mail address: vtlhang@dthu.edu.vn 